Proc. of the International Conference on Mechanochemistry and Mechanical Alloying, Kraków, Poland, June 22-26, 2014

\title{
"Mechanical Alloying-Compaction-Electroplating" as Technological Scheme for the Development of Nanocomposite Coatings
}

\author{
V. POPOV \\ National University of Science and Technology "MISIS", Moscow, Russia
}

\begin{abstract}
A novel technique of producing composite electrochemical coatings with non-agglomerated nanodiamond reinforcing particles uniformly distributed in the matrix was developed. The technique is intended for coatings to be applied by transferring material from the anode to the cathode. The anode is fabricated using the mechanical alloying method that enables a composite with the uniform distribution of non-agglomerated nanoparticles to be formed.
\end{abstract}

DOI: $10.12693 /$ APhysPolA.126.1005

PACS: 81.15.Pq, 81.05.Ni, 81.20.Ev

\section{Introduction}

Prospective way of metal electrodeposited coatings [1] properties improvement is application of nanomaterials for reinforcing metal matrix [2-4]. Almost immediately after their discovery, nanodiamonds $[5,6]$ began to be used for reinforcing electrochemical coatings $[7,8]$. The existing methods include the incorporation of diamond nanopowders (usually as suspensions) into the electrolyte. In this case, nanoparticles are in an agglomerated state. In electrochemical application of coatings, agglomerates are in practice not split; large agglomerates are "screened out" and retained in the electrolyte because primary nanoparticles and fine agglomerates are more readily transported to the coating application site. Thus, the existing methods enable the formation of so-called diamond-cluster coatings [9]. The main drawback of such coatings is that agglomerates of nanodiamond particles (clusters) do not possess high strength and break down under the action of cyclic loads, which leads to stress raisers and breakdown of the coating [10]. Thus, agglomeration of nanoparticles is the main obstacle for extensive introduction of nanocomposite electrodeposited coatings into practice. This work is aimed to refine techniques of applying composite coatings with nanodiamond reinforcing particles.

To eliminate the above-mentioned drawback, it is proposed to use the effect of disintegrating nanoparticle agglomerates in mechanical alloying [11-14] to produce coatings with separate reinforcing nanoparticles. That is, to fabricate the anode by mechanically alloying composite components and consolidating produced granules into a compact material and then to use the anode for applying the coating by the electrochemical method [15]. The technique is intended only for those kinds of coatings (copper, nickel etc.) that can be applied by dissolving the anode.

\section{Materials and methods}

A copper-based composite material with 20 vol.\% nanodiamond reinforcing particles was chosen for studies. Commercially available copper, grade $\mathrm{M} 0$, and nanodiamonds produced by Kombinat "Elektrokhimpribor" (Russia) were used. Primary nanodiamond particles 4-6 $\mathrm{nm}$ in size (Fig. 1a) were combined into agglomerates of up to $50-100 \mu \mathrm{m}$ (Fig. 1b) [16, 17].

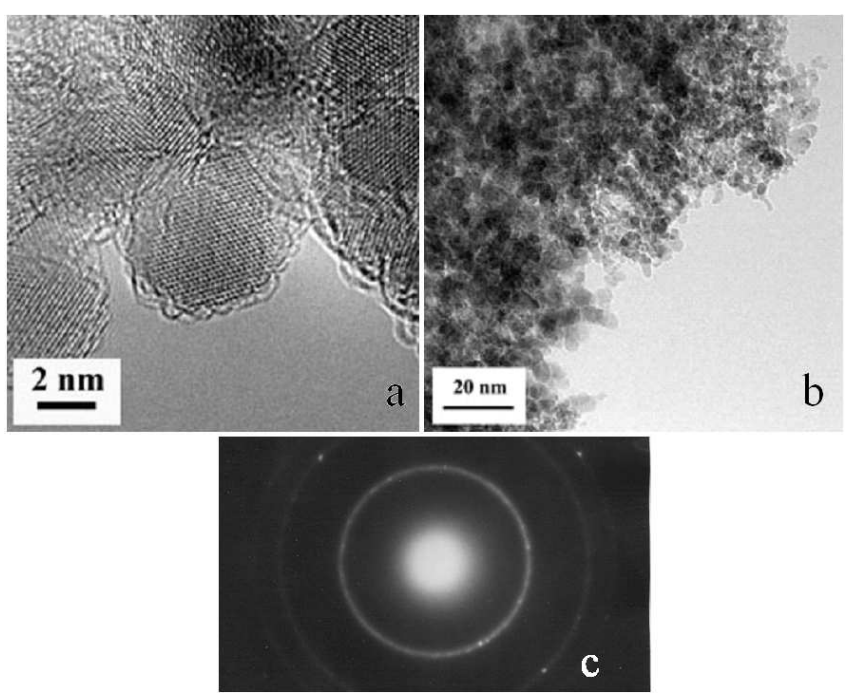

Fig. 1. Nanodiamonds of detonation synthesis: (a) initial prime nanodiamond particle (TEM), (b) agglomeration of nanodiamonds (TEM), (c) electron diffraction patterns from nanodiamonds (produced with TEM).

Mechanical alloying was carried out in a Retsch PM400 planetary mill in an argon atmosphere, without using surfactants, in sealed steel grinding jar containers of $500 \mathrm{ml}$ nominal volume. The technological milling tool was chromium steel balls $12 \mathrm{~mm}$ in diameter. The following technological conditions were chosen: (i) the ratio 
of the weight of the balls to that of the treated mixture, (7-10):1; (ii) the rotation velocity of the grinding jar containers around the common axis (the rotation rate of the carrier), $300 \mathrm{rpm}$; (iii) the grinding jar containers were cooled with air during the operation; (iv) to prevent strong overheating, the mill was stopped for 5 min after each $10 \mathrm{~min}$ of operation; the treatment time (without time for stops) was $3-5 \mathrm{~h}$.

The granules produced by mechanical alloying were compacted into bulk material using a hydraulic press. At first, composite granules were put in a special pressmould, the mould was covered by a removable punch, and the granules were pressed at room temperature under $600 \mathrm{MPa}$ pressure. Then the press-mould with the pressed granules closed by the removable punch was heated up to $600^{\circ} \mathrm{C}$, put in the integrated with hydraulic press thermostat heated up to $600^{\circ} \mathrm{C}$ and kept during 30-40 min under $300 \mathrm{MPa}$ pressure. Granules were compacted into rods $10 \times 10 \times 80 \mathrm{~mm}^{3}$ in size, which were used as anodes. The electrochemical coating was applied in a bath with an electrolyte containing $\mathrm{CuSO}_{4} \cdot 5 \mathrm{H}_{2} \mathrm{O}$, $80 \mathrm{~g} / \mathrm{l}$, and $\mathrm{H}_{2} \mathrm{SO}_{4}, 100 \mathrm{~g} / \mathrm{l}$, at a cathode current density of $10 \mathrm{~mA} / \mathrm{cm}^{2}=1 \mathrm{~A} / \mathrm{dm}^{2}$. Duration of the electrodeposition process was $3 \mathrm{~h}$. The structures and properties of developed materials were studied by optical microscopy, transmission (TEM) and scanning electron microscopy (SEM) and by the microhardness test. Optical microscopy was carried out at an Axiovert 200M MAT microscope (Carl Zeiss, Germany). The microhardness was determined by the Vickers method on a 402MVD microhardness tester (Wilson \& Wolpert) at a load of $10 \mathrm{gf}$, holding time $10 \mathrm{~s}$ (number of measurements, 10).

\section{Results and discussion}

Studies of the application of composite coatings were carried out according to the following scheme: mechanical alloying to produce a uniform distribution of nonagglomerated nanodiamond particles in the copper matrix - compaction to produce compacts in the form of anodes - electrodeposition of composite coatings by the electrolytic transfer of material from the anode to the cathode. Figure 2 demonstrates the appearance of composite granules under different magnifications.

It is seen that the chosen process regimes of mechanical alloying made it possible to produce composite granules with uniform distribution of non-agglomerated nan-

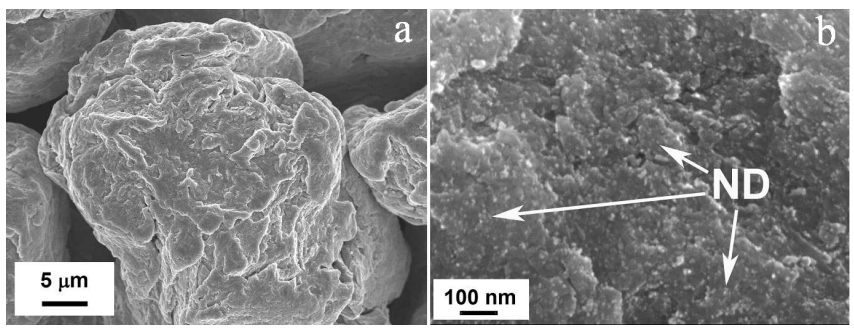

Fig. 2. Composite granules under different magnifications (SEM).

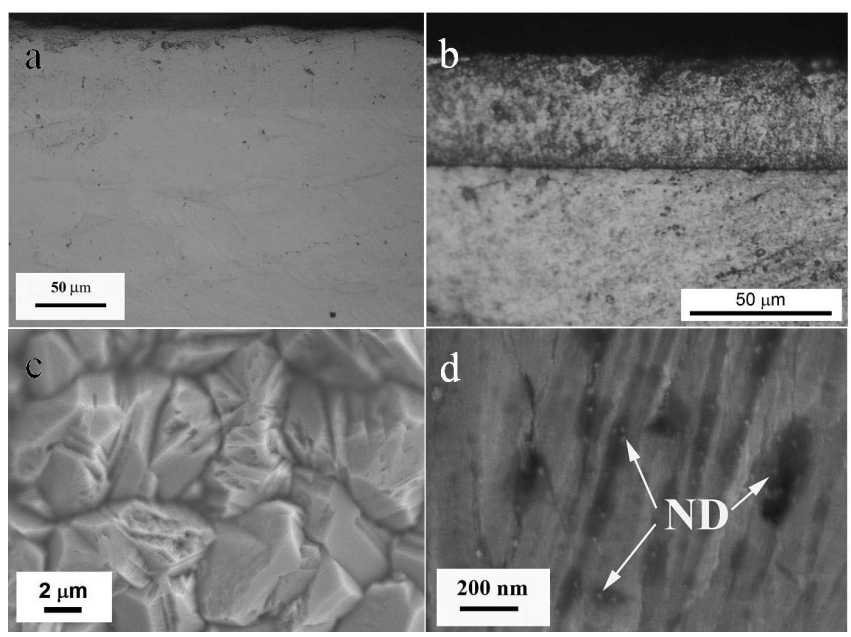

Fig. 3. Electrochemical composite coating: : (a) and (b) - cross-section images produced by optical microscopy before (a) and after (b) the etching, (c) planar view of coating (SEM), (d) nanodiamonds in the cross-section (SEM).

odiamond particles in the copper matrix. There is no increased oxidation of the matrix material, which is possible [18] through wrongly chosen process regimes. Following the compaction of the anode from these granules, the composite coating was electrodeposited; herewith, nanoparticles were transferred in the process of electrolysis in the electrolyte medium. The anode is dissolved, solvated nanoparticles are transferred to the cathode, where under the action of current the nanostructured coating is being formed. In this variant, during their transfer from the anode to the cathode the reinforcing nanosized particles are protected by the medium from agglomeration.

A 38-41 $\mu \mathrm{m}$ thick coating was obtained. Surface and the cross-sections of the coating were studied with optical and scanning electron microscopes (Fig. 3). The coating contains separate nanodiamond particles uniformly distributed in the matrix. There are no discontinuity defects. The coating has a high adhesion to the base.

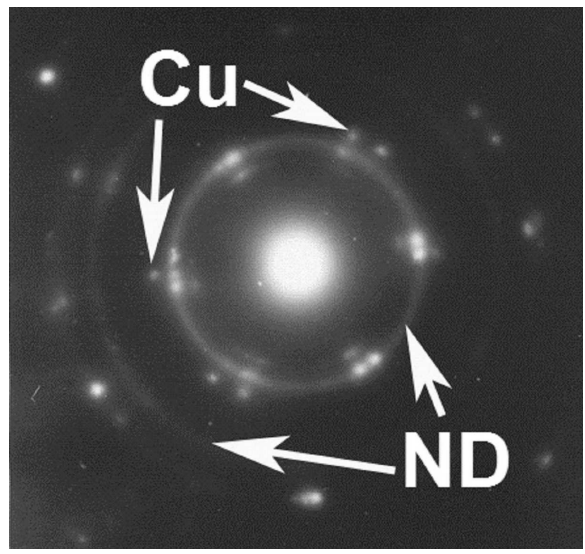

Fig. 4. Electron diffraction patterns from composite coating: point-reflexes are from copper and circles are from nanodiamonds (produced with TEM). 
Figure 4 shows electron diffraction patterns from composite coating included point reflexes and circles. Point reflexes are from copper big grains (Fig. 3c) and circles are from nanodiamonds (see Fig. 1c). It is proof that nanodiamonds are included in composite without any change of structure.

The composite coating microhardness measured in cross-sections was $105 \pm 5 \mathrm{HV} 0.01$ (microhardness of copper coating without reinforcing particles equals $45-$ $65 \mathrm{HV} 0.01$ ).

Thus, the proposed method was shown to enable the formation of an electrochemical composite coating with nanosized reinforcing particles. The method can be assumed to be efficient with other metals for the matrix, for instance, nickel, gold and others.

\section{Conclusions}

A process scheme of producing a composite coating was developed, including (a) mechanical alloying to produce a uniform distribution of non-agglomerated nanodiamond particles in the copper matrix; (b) compaction to produce compacts in the form of anodes; (c) electrodeposition of composite coatings by the electrolytic transfer of material from the anode to the cathode.

The studies performed show the following:

- mechanical alloying allows obtaining complete disintegration of nanodiamond agglomerates and uniform distribution of diamond nanoparticles in metal matrix;

- electrochemical method of coating application allows transferring composite material from anode to cathode;

- no nanoparticle agglomeration is observed during coating application, i.e. the metal matrix of the coating is reinforced by evenly distributed non-agglomerated nanodiamonds;

- good composite coating adhesion to the substrate, no defects either in the coating body or in the area of coating contact with the substrate.

\section{Acknowledgments}

These investigations were carried out with partial support from the Russian Foundation for Basic Research, project \#12-08-00185. The author is grateful to A.S. Prosviryakov, B.B. Chernov, I.M. Karnaukh, and A.L. Chuvilin for technical assistance in research.

\section{References}

[1] R. Karslioğlu, M. Uysal, A. Alp, H. Akbulut, Acta Phys. Pol. A 123, 424 (2013).

[2] H. Gül, M. Uysal, H. Akbulut, A. Alp, Acta Phys. Pol. A 125, 254 (2014).

[3] V.N.Tseluikin, Protect. Met. Phys. Chem. Surf. 45 , 312 (2009).

[4] Ajaya Kumar Pradhan, Siddhartha Das, J. Alloys Comp. 590, 294 (2014).

[5] V.V. Danilenko, Phys. Solid State 46, 581 (2004).

[6] V.A. Popov, A.V. Egorov, S.V. Savilov, V.V. Lunin, A.N. Kirichenko, V.N. Denisov, V.D. Blank, O.M. Vyaselev, T.B. Sagalova, J. Surf. Investig. Xray Synchr. Neutron Techn. 7, 1034 (2013).

[7] P. Cojocaru, A. Vicenzo, P.L. Cavalotti, J. Solid State Electrochem. 9, 850 (2005).

[8] V.P. Isakov, A.I. Lyamkin, D.N. Nikitin, A.S. Shalimova, A.V. Solntsev, Protect. Met. Phys. Chem. Surf. 46, 578 (2010).

[9] I.V. Ruban, Z.A. Godzhaev, V.M. Ruban, S.D. Zaytsev, Traktory i selskokhozyaystvennye mashiny (Tractors and Agricultural Machines) 7, 53 (2008) (in Russian).

[10] V.A. Popov, E.V. Nikitin, V.A. Zaytsev, D.V. Matveev, Tekhnologiya metallov (Technology of Metals) 3, 168 (2010) (in Russian).

[11] C. Suryanarayana, N. Al-Aqeeli, Prog. Mater. Sci. 58, 383 (2013).

[12] H. Gencer, N.E. Cengiz, V.S. Kolat, T. Izgi, S. Atalay, Acta Phys. Pol. A 125, 214 (2014).

[13] V.A. Popov, B.B. Chernov, A.M. Nugmanov, G.P. Schetinina, Fuller. Nanotubes Carbon Nanostruct. 20, 455 (2012).

[14] B. Ertuğ, B.N. Çetiner, G. Sadullahoğlu, H. Gökçe, Z.E. Erkmen, M.L. Öveçoğlu, Acta Phys. Pol. A 123, 188 (2013).

[15] V.A. Popov, B.B. Chernov, A.S. Prosviryakov, V.V. Cheverikin, I.I. Khodos, J. Biskupek, U. Kaiser, J. Alloys Comp., in press, corrected proof, available online 29 January 2014.

[16] P.Ya. Detkov, V.A. Popov, V.G. Kulichikhin, S.I. Chukhaeva, in: Molecular Building Blocks for Nanotechnology: From Diamondoids to Nanoscale Materials and Applications (Topics in Applied Physics, Vol. 109, Eds. G.A. Mansoori, T.F. George, G.P. Zhang, L. Assoufid, Springer, New York 2007, p. 29.

[17] V.A. Popov, in: Nanocomposites: Synthesis, Characterization and Applications, Ed. X.Y. Wang, Nova Science Publ., Inc., New York 2013, p. 369.

[18] V. Popov, in: Proc. Int. Conf. "Mechanics of Nano, Micro and Macro Composite Structures", Politecnico di Torino, 2012, Italy. 